\title{
A rare case of thyroid angiosarcoma - a diagnosis not to be missed.
}

\author{
Dimitris Kehagias $^{1}$, Eirini Kostopoulou ${ }^{1}$, and Konstantinos Panagopoulos ${ }^{1}$ \\ ${ }^{1}$ University General Hospital of Patras
}

June 30, 2020

\begin{abstract}
Primary thyroid angiosarcoma (TAS) is a rare mesenchymal tumor with poor prognosis, that should be differentiated from anaplastic thyroid carcinoma. We present the 60th case of this rare tumor and highlight the importance of early diagnosis, undelayed surgery and complementary radiation in order to improve prognosis and overall survival.
\end{abstract}

\section{Background:}

Primary thyroid angiosarcoma (TAS) is the most common of the mesenchymal thyroid tumors, which are extremely rare and occur in a rate less than 1\% [1], [2]. They show a high prevalence in Alpine areas, which is probably related to iodine deficiency in the areas and the presence of multinodular goiter [3], [4]. Additional predisposing factors are reported in the literature, such as exposure to radiation and vinyl chloride [5], [6]. It occurs mainly in adults 70 years of age, with a higher rate in women (4.5:1 ratio) [3]. The clinical manifestations of TAS are non-specific and vary depending on the location, size, extension and metastases. It generally presents as an invasive mass in the cervix causing symptoms of obstruction due to its rapid growth, such as shortness of breath, hoarseness and dysphagia. Fever, anemia and leukocytosis are often observed in the context of paraneoplastic syndrome [7]. TAS is an aggressive neoplasm with poor prognosis and resulting in death within 6-9 months due to premature metastases to lymph nodes, lungs, bones and soft molecules [8], [9]. TAS is reported to be a very rare entity, with a recent literature review recording 59 cases [8]. Herein we describe the $60^{\text {th }}$ case of a 69 -year-old female patient from Greece.

\section{Case presentation}

A 69-year-old female patient with a known for years multinodular goiter, presented with a 3-month history of fever up to $38.5^{\circ} \mathrm{C}$, night sweating and tenderness in the palpation of the right lobe of the thyroid gland. She also reported fatigue and a 10-kg weight loss over the past 1 month. Fine needle aspiration (FNA) was performed in order to exclude acute thyroiditis, but the cytological picture was compatible with a low-differentiation neoplasm, most likely metastatic adenocarcinoma. The patient was submitted to chest, abdominal and brain computed tomography (CT) scan, a mammography and gastrointestinal endoscopic examination (colonoscopy, gastroscopy), which all revealed no pathological findings. A thyroid scintigraphy with Technetium-99m was also performed, which identified a large multinodular goiter and substernal extension to the right, causing displacement of the trachea. Immediately after, she was taken to the operating theatre where a nodule of hard composition and red-gray coloured surface of about $5 \mathrm{~cm}$ in the right lobe of the thyroid and two nodules of the same appearance in the sternohyoid muscle, $2 \mathrm{~cm}$ below the hyoid bone, were identified (Figure 1). Total thyroidectomy and resection of the nodules in the sternohyoid muscle were performed and the patient was discharged on the 3rd postoperative day without any complications.

The histological examination revealed unspecified borders of the nodules and areas of high mitotic index, necrosis and hemorrhage. A high-grade neoplasia was confirmed with large epithelioid cells forming solid foci or lining the wall of cystic spaces filled with red blood cells. An infiltrative pattern was observed, 
albeit without rupture of the thyroid capsule, but with infiltration to the strap muscles, especially in the right sternohyoid muscle. The tumor immunostained for vascular markers (vimentin, CD31 and CD34), whereas epithelial differentiation markers, such as cytokeratins AE1/AE3 were mildly expressed or others, thyreoglobulin and calcitonin, were totally negative. Free surgical margins were detected and no lymph node metastases were recognized.

The diagnosis of thyroid angiosarcoma with extra-thyroid extension to the sternohyoid muscle was established. The patient was subsequently under the oncology surveillance and received 25 sessions of radiotherapy. Chemotherapy with paclitaxel was also suggested, but the patient refused further treatment. She died of the disease 9 months post-surgery.

\section{Discussion and Conclusions}

TAS is a rare and aggressive mesenchymal tumor, which has been the subject of controversy, especially when it comes to it being differentiated from anaplastic thyroid carcinoma. For years it was considered a vascular mutation of anaplastic carcinoma and not a true sarcoma. The first recorded case of angiosarcoma was in 1986, when immunohistological techniques confirmed the endothelial origin of the tumor [10]. Primary angiosarcoma was only recognized as a distinct entity in the WHO classification in 2013 [11].

Histologically, TAS is characterized by areas of extensive necrosis and bleeding with the presence of endothelial cells. The neoplastic cells are large with a high mitotic index and tumor necrosis prevails. In addition to cytokeratin, neoplastic cells also express endothelial markers, which allow differentiation from anaplastic carcinoma. In a bibliographic report of 23 patients, positive immunohistochemical staining for endothelial markers or cytokeratin was observed in all cases [12]. Specifically, CD31 was positive in all 19 patients tested, while CD34 was positive in 7 of 16 cases (44\%). Factor VIII was positive in 20 of 23 patients (87\%) and cytokeratin in 22 of $23(96 \%)$. Therefore, cytokeratin, which is also expressed in anaplastic carcinomas, does not help in the differential diagnosis from anaplastic carcinoma, as opposed to CD31 and factor VIII, which in combination with tumor morphology, essentially determine the endothelial origin of the tumor [12]. Another essential characteristic of TAS is the negative expression of thyroglobulin, which differentiates angiosarcoma from anaplastic carcinoma, in which thyroglobulin is mildly expressed [13], [14].

TAS is a particularly aggressive tumor with poor prognosis, as confirmed from the latest literature review that cites all the known 59 cases, in $89.3 \%$ of which death occurred within 9 months [8]. Death occurs due to rapid metastases to lymph nodes, lungs, bones and soft molecules [3]. Infiltration of the trachea and esophagus are also common. Capsule infiltration and distant metastases represent the most negative prognostic factors, whereas a better prognosis is expected when TAS is limited to the thyroid gland and is combined with aggressive treatment [15], [16].

Due to the rarity and the small number of TAS cases, no gold standard of treatment has been established. Undelayed radical surgery along with complementary radiation seems to improve prognosis and overall survival and is, therefore, recommended as the best approach based on the data so far [17]. Sarcomas should be approached by many specialties and if it is possible, they should be treated at a referral centre [18]. Chemotherapy may also have a local and systemic effect on disease control [19], nonetheless, more studies are needed to determine the role of chemotherapy [20]. Radioactive iodine has no place in the treatment of vascular sarcoma, since the cells are not of thyroid origin and new drugs, such as anti-VEGF agents [21] and paclitaxel [22], have been tested without satisfactory results.

In conclusion, the present case is the $60^{\text {th }}$ to be reported in the literature. Raising awareness to clinicians about this tumor is important so that early diagnosis and treatment increase the chances of survival. In addition, recording new cases of this rare neoplasm that provide additional data on treatment, the course of the disease and the final outcome, may help identify the best treatment approach in order to improve prognosis and overall survival.

\section{Declarations}

Funding: No funding was received. 
Data Availability Statement: Data sharing is not applicable to this article, as no datasets were generated or analyzed during the current study.

Author's contributions: Dr Dimitrios Kechagias wrote the first draft of the manuscript, participated in the surgery performed to the patient and contributed to the conception of the manuscript. Eirini Kostopoulou revised the manuscript, performed a thorough literature review and completed the writing. Professor Panagopoulos was the surgeon in charge for this case, he performed the surgery, contributed to the conception and gave the final approval of the current version to be published.

Consent for publication: Consent for the publication of all associated data and the accompanying image was provided from the patient's next of kin, because the patient was deceased.

Competing interests: The authors declare that they have no competing interests.

\section{References}

[1] John Goldblum, Enzinger and Weiss's Soft Tissue Tumors, St Louis: Elsevier, 2019.

[2] Surov A, Gottschling S, Wienke A, Meyer HJ, Spielmann RP, Dralle H. Primary Thyroid Sarcoma: A systematic review. Anticancer Res. 2015:35:5185-91.

[3] WHO Classification of Tumours of Endocrine Organs. Fourth Edition, Ed. Lloyd RV. Geneva: WHO, 2017.

[4] Kurman R. Atlas of tumor pathology, Washington DC: Armed forces Institute of Pathology, Washington, DC, 1992.

[5] Meis-Kindblom JM, Kindblom LG. Angiosarcoma of soft tissue: a study of 80 cases. Am J Surg Pathol. 1998;22:683-97.

[6] Collini P, Barisella M, Renne SL, Pizzi N, Mattavelli D, Stacchioti S, et al. Epithelioid angiosarcoma of the thyroid gland without distant metastases at diagnosis: report of six cases with a long follow-up. Virchows Arch. 2016;469:223-32.

[7] De Felice F, Moscatelli E, Orelli S, Bulzonetti N, Musio D, Tombolini V. Primary thyroid angiosarcoma: A systematic review. Oral Oncol. 2018;82:48-52.

[8] Huang SH, Wu SC. Primary Angiosarcoma of the Thyroid in an Asian Woman: A Case Report with Review of the Literature. Case Rep Pathol. 2020: 9068506. doi: 10.1155/2020/9068506.eCollection 2020.

[9] Marina M, Corcione L, Serra MF, Ferri T, Silini EM, Ceresini G. Primary Epithelioid Angiosarcoma of the Thyroid in a Patient Occupationally Exposed to Radiations. Front Endocrinol (Lausanne). 2018;9:577.

[10] Chan YF, Ma L, Boey JH, Yeung HY. Angiosarcoma of the thyroid. An immunohistochemical and ultrastructural study of a case in a Chinese patient. Cancer, 1986;57:2381-8.

[11] WHO classification of soft tissue tumors: an update based on 2013 edition, ed. Jo VY, 2014.

[12] Rotellini M, Vezzosi V, Bianchi S. Epithelioid Angiosarcoma of the Thyroid: Report of a Case from an Italian Non-Alpine Area and Review of the Literature. Endocr Pathol. 2015;26:152-6.

[13] Petronella P, Scorzelli M, Luise R, Iannaci G, Sapere P, Ferretti M, et al. Primary thyroid angiosarcoma: an unusual localization. World J Surg Oncol. 2012;10:73.

[14] Binesh F, Akhavan A, Navabii H, Dadfarnia MH, Zand V. Primary angiosarcoma of the thyroid gland in an young Iranian woman. BMJ Case rep. 2011;2011:bcr0320114042.

[15] Maiorana A, Collina G, Cesinaro AM, Fano RA, Eusebi V. Epithelioid angiosarcoma of the thyroid. Clinicopathologic analysis of seven cases from non Alpine areas. Virchows Arch. 1996;429:131-7. 
[16] Kalitova P, Pizak J, Kodet R, Astl J. Angiosarcoma of the thyroid. Eur Arch Othorhinolaryngol. 2009;266:903-5.

[17] Mattavelli D, Miceli R, Radaelli S, Mattavelli F, Cantu G, Barisella M, et al. Head and Neck Soft Tissue Sarcomas: Prognostic Factors and Outcome in a Series of Patients Treated at a Single Institution. Ann Oncol. 2013;24:2181-9.

[18] Casali PG, Abecassis N, Aro HT, Bauer S, Biagini R, Bielack S, et al. Soft tissue and visceral sarcomas: ESMO Clinical Practice Guidelines for diagnosis, treatment and follow-up. Ann Oncol. 2018;29:iv51-iv67.

[19] Wiedermann JP, Sadeghi N, Baird-Howell M. Rapidly Growing Vascular Mass Within the Thyroid. JAMA Otolaryngol Head Neck Surg. 2016;142:397-8.

[20] Abraham JA, Hornicek FJ, Kaufman AM, Harmon DC, Springfield DS, Raskin KA, et al. Treatment and Outcome of 82 Patients With Angiosarcoma. Ann Surg Oncol. 2007;14:1953-67.

[21] Park MS, Ravi V, Araujo DM. Inhibiting the VEGF-VEGFR Pathway in Angiosarcoma, Epithelioid Hemangioendothelioma, and Hemangiopericytoma Solitary Fibrous Tumor. Curr Opin Oncol. 2010;22:3515 .

[22] Schlemmer M, Reichardt P, Verweij J, Hartmann JT, Judson I, Thyss A, et al. Paclitaxel in Patients With Advanced Angiosarcomas of Soft Tissue: A Retrospective Study of the EORTC Soft Tissue and Bone Sarcoma Group. Eur J Cancer. 2008;44:2433-6.

\section{Caption}

Figure 1. Angiosarcoma of the right thyroid lobe and nodules localised in the sternohyoid muscle.

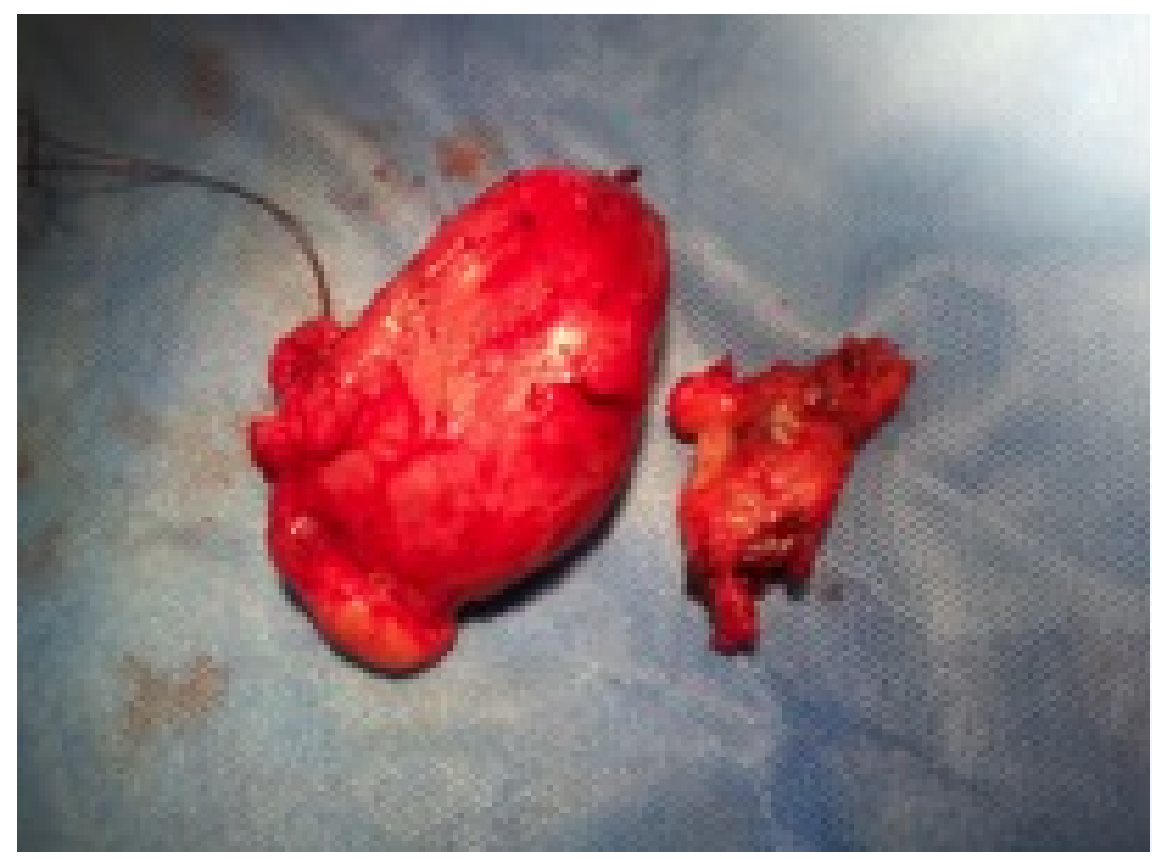

